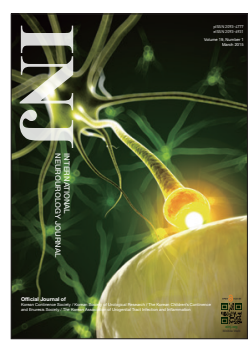

\title{
Urogynecology and Reconstructive Pelvic Surgery. 4th ed.
}

By Mark D. Walters, Mickey M. Karram. Philadelphia: Saunders; 2014. 688 pp. \$169.00. ISBN: 978-0323113779

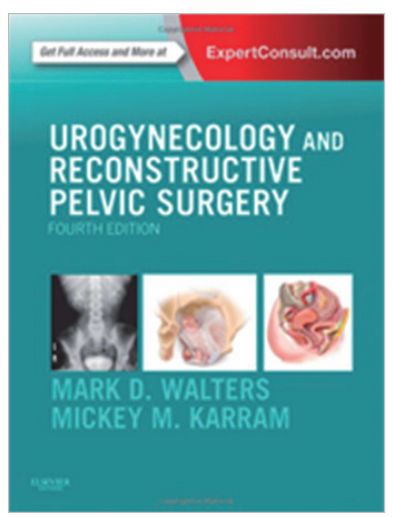

This is a truly comprehensive textbook that covers the whole spectrum of female pelvic medicine and reconstructive surgery (FPMRS). It starts with reviewing the history of female pelvic surgery, gynecology, and female urology. This is a nice setup for covering the latest evolutions in FPMRS such as female sexual dysfunction, robotic surgery for pelvic organ prolapse, and surgical management of detrusor compliance abnormalities. The basic science part includes chapters that are dedicated to the physiology of the pelvic muscles, vagina, and anorectum. In addition, the chapter on the psychosocial impact of pelvic floor disorders provide the reasons for trying to master this difficult to learn field. A welcome addition to this edition is the step-by-step instructions on regular and video urodynamics. But perhaps more important may be the two dedicated two chapters and interspersed review of possible surgical complications and their method of management. Four chapters are dedicated to fecal incontinence and defecation disorders, and the book ends with explaining on what to use in measuring outcomes and quality of life in pelvic floor research. This book should be seriously considered by anyone involved in FPMRS.

Jang Hwan Kim Department of Urology, Yonsei University College of Medicine

E-mail: jkim@yuhs.ac 